\title{
A comparison of platelet aggregation produced by seven compounds and a comparison of their inhibitors ${ }^{1}$
}

\author{
J. R. O'BRIEN \\ From the Portsmouth and Isle of Wight Area Pathological Service
}

SYNOPSIS The aggregating effects of adenosine diphosphate, thrombin, 5-hydroxytryptamine, tryptamine, adrenaline and noradrenaline, and tri-ethyl tin have been carefully compared. The first three compounds in some circumstances produce remarkably similar effects although there are important differences. The kinetics of aggregation induced by adrenaline (and noradrenaline) are quite different and the tri-ethyl tin effects are different again. Anti-serotonins specifically inhibit 5-hydroxytryptamine and the anti-adrenaline drug phentolamine specifically inhibits the effects of the catecholamines.

Experiments presented suggest but do not prove that aggregation produced by all these compounds is accompanied by the liberation of diphosphate from the platelets and that platelet triphosphate may be converted to diphosphate. How these different compounds all produce this effect is discussed. Either the presence of diphosphate or the action of a triphosphatase might be the immediate cause of aggregation if there is a single final common cause. The anti-adrenaline phentolamine prolongs the bleeding time, so adrenaline or noradrenaline may be involved in platelet phenomena in haemostasis.

It has now been reported that platelet aggregation can be brought about by thrombin, adenosine diphosphate (Gaarder, Jonsen, Laland, Hellem, and Owren, 1961), tri-ethyl tin (O'Brien, 1963a), and by noradrenaline and 5-hydroxytryptamine (Mitchell and Sharp, 1964). It is not known what forces and mechanisms are involved in platelet-to-platelet adhesion, although it has been shown that thrombin liberates the diphosphate (Grette, 1962) and it has been suggested that tri-ethyl tin may liberate disphosphate which thus might form a final common pathway (O'Brien, 1963b). A detailed comparison of the effects of all these compounds and their inhibitors was urgently needed. How do so many different compounds all produce the same remarkable effect?

\section{METHODS}

MEASUREMENT OF PLATELET AGgREgation Freshly collected pooled citrated or heparinized (final concentration

'Some of the material presented here has been summarized in Nature $(200,763,1963)$ and in two lectures which are in the press: 'The International Committee for the Nomenclature of Blood Clotting Factors' (Thromb. et Diath. Haem.) and 'The Proceedings of the IX Congress of the European Society of Haematology', S. Karger.

Received for publication 3 October 1963.
$4 \mathrm{u}$. per ml.) human platelet-rich plasma containing about 400,000 platelets per c.mm. was kept at $15^{\circ} \mathrm{C}$. and was normally warmed to $37^{\circ} \mathrm{C}$. for five minutes before use. Each pool was used within six hours of collection. Two millilitres were placed in a cuvette, and stirred at a constant optimal speed by a magnetic stirrer. The cuvette, kept at $37^{\circ} \mathrm{C}$. was put in an EEL titrometer (a photocolorimeter) and the light transmission through the cloudy suspension was recorded in arbitrary units on a Honeywell recorder (O'Brien, 1962). A similar method has been carefully evaluated by Born and Cross (1963b). Thus a second by second record of the degree of dispersion of the platelets was obtained. Normally, the plasma was stirred for a sufficient period to ensure that no spontaneous aggregation would occur, and then sufficient aggregating compound dissolved in $0.1 \mathrm{ml}$. of barbitonebuffered saline was added to give the final concentrations mentioned in the text. The magnitude of the response of different batches of plasma to the same dose of an aggregating agent, and especially to inhibitors, differed somewhat, so each set of comparisons had to be carried out on the same day and the slightly differing results of different days were averaged.

TEST OF INHIBITORS in vivo Sterile solutions of the inhibitors were injected intradermally into the human forearm in $0.1 \mathrm{ml}$. amounts by a tuberculin syringe. 
Usually five different compounds, always including saline as a control, were injected in differing orders down the arm. With a sphygomanometer cuff inflated on the upper arm to $40 \mathrm{~mm}$. $\mathrm{Hg}$ (Borchgrevink, 1960) cuts $0 \cdot 8 \mathrm{~cm}$. long were made across the injection sites, and the bleeding times were estimated.

\section{RESULTS}

TIME PATTERN OF AGGREGATION AND DOSE RESPONSE These are described for each substance.

Adenosine diphosphate Increasing concentrations of this diphosphate were added to aliquots of the same platelet-rich plasma and Fig. 1 records a typical family of curves. It will be seen that aggregation begins at once and that with increasing concentrations the initial slope increased indicating more rapid aggregation. The initial slopes of these curves, which are almost linear, are proportional to the log of the dose of diphosphate added. With low concentrations, but over at least a five-fold range, aggregation stops after about 40 seconds, the inactivation time, then disaggregation occurs. The added diphosphate must have been inactivated or is no longer available since it can be shown that these platelets at this time will reaggregate if a further dose of diphosphate is added. Since the inactivation time is the same when the added diphosphate concentration is increased from $3 \mathrm{M} \times 10^{-8}$ to $\mathrm{M} \times 10^{-7}$ it can be concluded that higher concentrations are

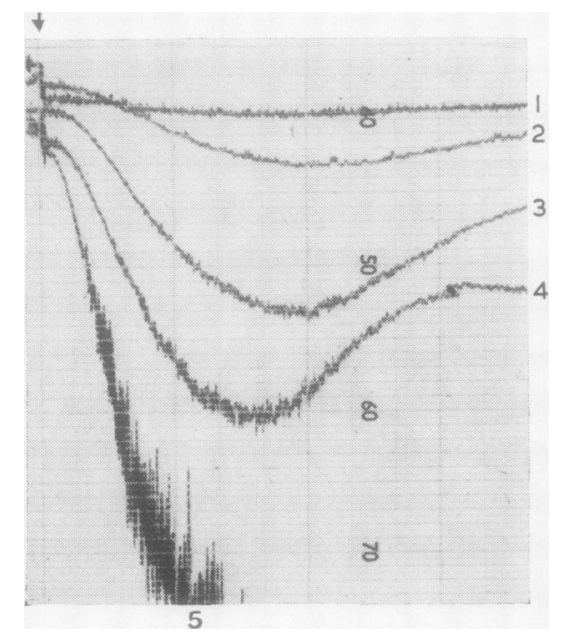

FIG. 1. One major vertical division represents 30 seconds. An increase in transmission (down the tracing) indicates aggregation. At zero time $(\downarrow)$ sufficient adenosine diphosphate in $0.1 \mathrm{ml}$. of saline was added to aliquots of the same platelet-rich plasma to give in tracing (I) a final concentration of $M \times 10^{-8}$, in tracing (2) $3 M \times 10^{-8}$, in tracing (3) $6 M \times 10^{-8}$, in tracing (4) $M \times 10^{-7}$, and in tracing (5) $4 M \times 10^{-7}$. inactivated considerably faster. A further importan consequence of the roughly constant time taken to. inactivate varying concentrations of diphosphate is that the maximum degree of aggregation observeff for any particular dose is proportional to the initiat slope and hence it is also proportional to the origina. dose. Thus to characterize the effect of any giver dose it is, in practice, more convenient to measure the total change in transmission than the initiat slope.

The approximate concentrations required to pro $=$ duce minimal and maximal aggregation (both poorly defined criteria) for adenosine diphosphate and othes? compounds is given in Table $\mathrm{I}$. The effect of adding a second equal dose of diphosphate and other com $\vec{v}$ pounds after the platelets have disaggregated oiw reached a plateau after a first dose, is given in Table III and illustrated in Figure 2.

\section{TABLE I}

\begin{tabular}{lll} 
Dggregating & DOSE RESPONSE RANGE \\
Compound & $\begin{array}{l}\text { Minimum Aggregation } \\
\text { Produced by }\end{array}$ & $\begin{array}{l}\text { Maximum Aggregation } \\
\text { Produced by }\end{array}$ \\
\hline
\end{tabular}

\begin{tabular}{|c|c|c|}
\hline A.D.P. & $3 \mathrm{M} \times 10^{-8}$ & $3 \mathrm{M} \therefore 10^{-6}$ \\
\hline Thrombin & U 0.03 & U $\times 5.0$ \\
\hline 5-H.T. & $2 \mathrm{M} \therefore 10^{-7}$ & ${ }^{1} 5 \mathrm{M} \therefore 10^{-6}$ \\
\hline Adrenaline & $5 \mathrm{M} \times 10^{-8}$ & $5 \mathrm{M} \therefore 10^{6}$ \\
\hline Noradrenaline & $5 \mathrm{M} \times 10^{-7}$ & $5 \mathrm{M} \times 10^{-5}$ \\
\hline Tri-ethyl tin & $2 \mathrm{M} \times 10^{-6}$ & ${ }^{2} \mathrm{M} \therefore 10^{-4}$ \\
\hline
\end{tabular}

${ }^{1}$ When the concentration is increased above this level the response $\overrightarrow{\bar{D}}$ decreases progressively.

2Only the delay period alters throughout this range.

Thrombin Only a small range of thrombin concentrations could be studied because with high? concentrations rapid clotting interfered with aggrega-o tion. It is shown in Fig. 2 that after the addition of thrombin there is a delay of about 10 seconds beforeo aggregation occurs; thereafter the time pattern of $₹$ aggregation and disaggregation is identical when음 produced by carefully selected equally effective doses of adenosine diphosphate, thrombin, and 5-hydroxy-을 tryptamine. A family of curves for the different possible concentrations of thrombin was otherwise. similar to the curves for adenosine diphosphate and $N_{0}$ the log-dose slope relationship was again evident. $A_{\omega}^{N}$ second dose of thrombin added after disaggregation again produced aggregation after a further 10 -second delay (Fig. 2).

5-Hydroxytryptamine A concentration of $5 \mathrm{M} \times \stackrel{\mathscr{P}}{?}$ $10^{-8}$ produced minimal aggregation and then dis- $T$ aggregation. From $5 \mathrm{M} \times 10^{-7}$ to $5 \mathrm{M} \times 10^{-5} \mathrm{O}^{-}$ slightly more aggregation occurred. Still higher con- $\stackrel{\mathbb{D}}{\stackrel{D}{\mathbb{D}}}$ centrations had less effect. Thus the dose response $\mathbb{\mathbb { D }}$ curve is quite different from that of adenosine di- $\frac{\Omega}{\sigma}$ phosphate or thrombin. No concentration produced 


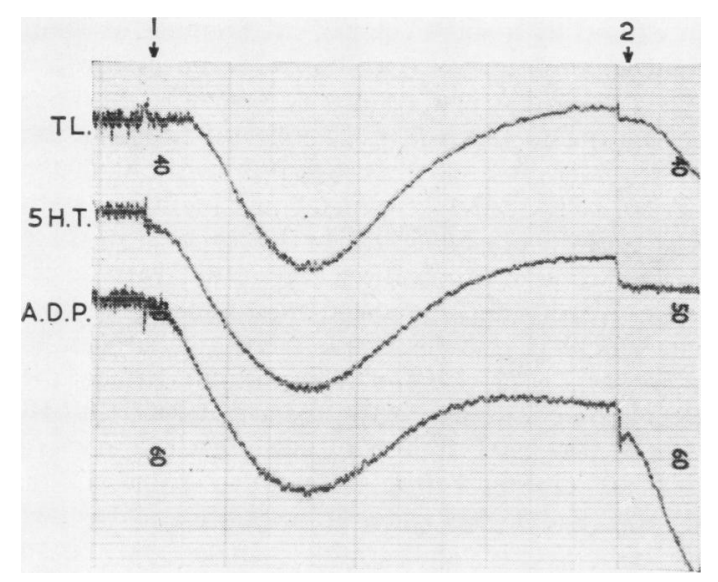

FIG. 2. One major vertical division represents 30 seconds. An increase in transmission (down the tracing) indicates aggregation. At $\downarrow^{1}$ in the tracing labelled Th, thrombin to give a final concentration of 0.06 units $/ \mathrm{ml}$. was added. At $\downarrow^{2}$ a second similar dose was added. At $\downarrow^{1}$ in the tracing labelled 5-HT 5-hydroxytryptamine was added, final concentration $5 \mathrm{M} \times 10^{-6}$ and a similar dose at $\downarrow^{2}$. At $\downarrow^{1}$ in the tracing labelled A.D.P, adenosine diphosphate final concentration $5 \mathrm{M} \times 10^{-7}$ was added and again at $\downarrow^{2}$.

more than a small degree of aggregation but the sequence of events plotted against time is again almost identical with the diphosphate (Fig. 2). A second dose of 5-hydroxytryptamine added at two or 30 minutes after a first dose produced no aggregation.

Tryptamine Tryptamine, like 5-hydroxytryptamine, over a wide range of doses caused the same degree of aggregation but at all concentrations the degree of aggregation was very small: 2 to 4 units of transmission compared with 5 to 12 units with 5-hydroxytryptamine. A second dose was ineffective.

Adrenaline and noradrenaline These two compounds behaved similarly in all respects, but an approximately tenfold higher concentration of noradrenaline was required to produce an equal effect. Inspection of a family of curves (Fig. 3 ) produced by adding increasing concentrations of adrenaline to aliquots of platelet-rich plasma shows that aggregation began immediately and that initially the slope depends on the concentration added, and, as with diphosphate, the slope was proportional to the log of the dose. With smaller doses, after about 70 seconds, a time that was constant over a considerable dose range, aggregation ceased, some disaggregation might occur and there was a plateau which, with very small doses of adrenaline, persisted (not illustrated). More often the plateau after a delay of from several seconds to five minutes, which was

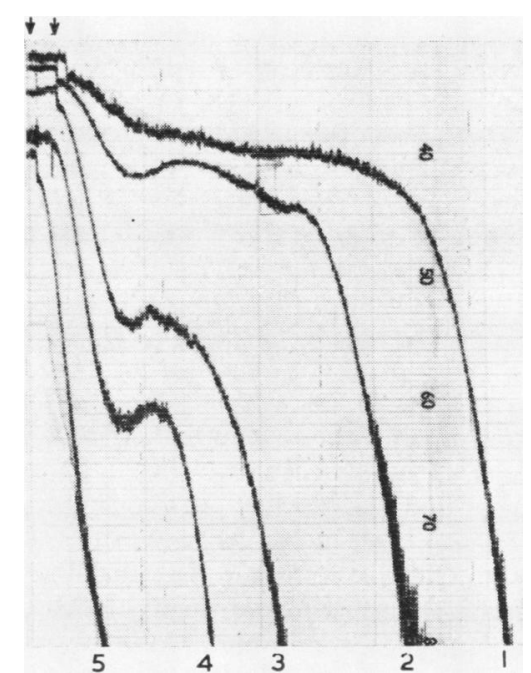

FIG. 3. One major vertical division represents two minutes. Between the arrows in tracing 1 sufficient adrenaline was added to an aliquot of platelet-rich plasma to give a final concentration of $2 \mathrm{M} \times 10^{-6}$; tracing 2 , final concentration $4 M \times 10^{-6}$; tracing $3, M^{-5}$; tracing 4, $2 M \times 10^{-5} ;$ tracing $5, M \times 10^{-4}$.

roughly dose dependent, was followed by a second period of increased and rapid aggregation. In this second period the rate of aggregation was always equally rapid, irrespective of the delay before it began or of the original concentration, and complete aggregation always occurred. A second dose of weak adrenaline added during a long delay period caused further aggregation. Isoprenaline was inactive and did not inhibit adrenaline-induced aggregation.

Tri-ethyl tin This phenomenon has already been described (O'Brien, 1963a and c). The effective range is narrow. Increasing the concentration within this range shortens the initial lag period of two to eight minutes during which no aggregation occurs. After this lag phase and almost irrespective of the initial concentration of tri-ethyl tin there is an explosive rapid period of aggregation which always proceeds to completion, that is, to maximal aggregation. Disaggregation occurs slowly or not at all.

Adenosine triphosphate There are a number of reports that the triphosphate inhibits aggregation induced by diphosphate but Mitchell and Sharp (1964) claim that the triphosphate can itself cause aggregation and O'Brien (1963c) has reported partial aggregation by this compound. If solutions made from the dry compound were tested at once they produced little or no aggregation. If these solutions were kept and tested during the next fortnight invariably an increased degree of aggregation occurred; a second dose added after disaggregation was always 
without effect. The inhibitory activity of this compound will be reported later.

TEMPORAL DECAY OF THE RESPONSE TO AGGREGATING COMPOUNDS Platelet-rich plasma incubated at $37^{\circ} \mathrm{C}$. in sealed containers failed to respond to adrenaline, noradrenaline, 5-hydroxytryptamine, and tri-ethyl tin after about 90 minutes. The plasma failed to respond to thrombin after 200 minutes and to adenosine diphosphate after three to four hours.

SYNERGISTIC EFFECTS OF AGGREGATING COMPOUNDS It was determined what concentrations of adenosine diphosphate, triphosphate, thrombin, 5-hydroxytryptamine, and adrenaline gave small similar degrees of aggregation, usually about 8 units of transmission. The experiment was then conducted on a Roman square principle, so that each possible pair of compounds was added simultaneously, including two doses of each of the compounds. Table II records the increase or decrease $(-)$, above or below the expected figure and represents the mean for three such experiments. Thus if thrombin alone gave a deflection of 5, and noradrenaline alone of 8 , and the two added together gave a deflection of 21 , then the difference between $(8+5)$ and 21 , i.e., 8 , is thought to indicate synergistic action.

THE EFFECT OF INHIBITORS in vitro The inhibitory concentration of a compound is here arbitrarily defined as the strength required to reduce the deflec- tion caused by a small dose of an aggregating com pound to slightly less than half. The concentration. of the aggregating compounds necessary to product aggregation of about 6 to 12 units of transmission? was first identified. Increasing concentrations of the inhibitor to be tested were mixed and incubated wit? aliquots of the plasma and then the response of these mixtures to the aggregating compound was deter mined. Adenosine, adenosine mono- and tri-phos phate, cocaine, and histamine were incubated withthe plasma at $37^{\circ} \mathrm{C}$. for five minutes before the aggregating compound was added. Reserpine, $\mathrm{UMI}_{\vec{\omega}}$ 491 and Ro 3/0837, and mono-iodo-acetate were added 30 minutes before testing. Reserpine, UMK 491 and Ro 3/0837, adenosine triphosphate, and adrenaline were made up freshly each day and kept ido the dark. The inhibitory concentrations of importanis compounds are reported in Table III. Histamine hadr no effect. Benadryl, at a concentration of $50 \mu \mathrm{g}$. peb $\mathrm{ml}$., inhibited the effect of 5-hydroxytryptamine and adrenaline; when used a little stronger it inhibite $\bar{\beta}$ all kinds of aggregation. Mono-iodo-acetate ank cocaine inhibited all aggregation, however produced $\overrightarrow{0}$ Phentolamine inhibited adrenaline quantitatively. Aक family of curves similar to Fig. 3 was constructed by் adding say $1,2,4$, and 8 units of adrenaline; then bs adding increasing amounts of phentalamine befores adding 8 units of adrenaline, curves similar to those्द of 4,2 , and 1 units of adrenaline alone, could be्ष obtained.

TABLE II

SYNERGISTIC EFFECTS

\begin{tabular}{|c|c|c|c|c|c|c|}
\hline \multirow[b]{2}{*}{ Compound } & \multicolumn{4}{|c|}{ SHINEKUIS IC ERTEC IS } & \multirow[b]{2}{*}{ Noradrenaline } & \multirow{2}{*}{ Adrenaline } \\
\hline & A.D.P. & Thrombin & 5-H.T. & A.T.P. & & \\
\hline $\begin{array}{l}\text { A.D.P. } \\
\text { Thrombin } \\
\text { 5-H.T. } \\
\text { A.T.P. } \\
\text { Noradrenaline } \\
\text { Adrenaline }\end{array}$ & $\begin{array}{l}1 \\
1 \\
4 \\
1 \\
5 \\
3\end{array}$ & $\begin{array}{l}1 \\
4 \\
1 \\
8 \\
5\end{array}$ & $\begin{array}{r}-2 \\
2 \\
6 \\
5\end{array}$ & $\begin{array}{l}2 \\
1 \\
0\end{array}$ & $\begin{array}{l}-1 \\
-1\end{array}$ & -1 \\
\hline
\end{tabular}

The figures reported are the increase in the transmission above the sum of the transmissions when the two compounds were added separately. $\vec{\supset}$

TABLE III

ACTION OF INHIBITORS ${ }^{1}$

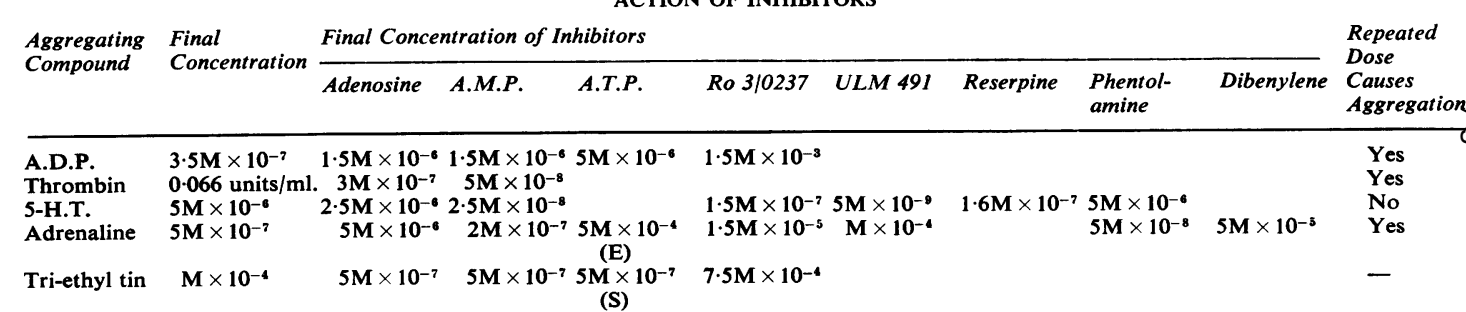

(S)

${ }^{1}$ Inhibition as defined in the text was caused by the inhibitors at the strengths shown. For tri-ethyl tin the strength of an inhibitor needed tœ prolong the lag phase is given: A.T.P. at the given strengths enhanced (E) the effect of adrenaline and shortened (S) the lag phase of tri-ethy $b$ tin. When high concentrations of the inhibitors had no effect on aggregation, the relevant space is left blank. 
The following experiment showed that when adrenaline causes platelet aggregation adenosine diphosphate is liberated. This might suggest that the release of diphosphate is responsible for aggregation induced by adrenaline. Adrenaline was added to $2.5 \mathrm{ml}$. stirred platelet-rich plasma to give a final concentration of $5 \mathrm{M} \times 10^{-6}$ and the mixture stirred till final complete aggregation was observed. This mixture was then rapidly centrifuged and $0.5 \mathrm{ml}$. of the supernatant was then added to another aliquot of platelet-rich plasma to which phentolamine, final concentration $5 \mathrm{M} \times 10^{-6}$, had previously been added. Marked aggregation immediately occurred. It could be inhibited by strong adenosine and the curve obtained was identical to that obtained with adenosine diphosphate. Adrenaline was added to platelet-poor plasma and treated identically; $0.5 \mathrm{ml}$. of this mixture was added to the platelet-rich plasma-phentolamine mixture and no aggregation occurred. The platelet-rich plasma was frozen and thawed twice and $0.5 \mathrm{ml}$. of the supernatant after centrifugation was added to a phentolamine plateletrich plasma mixture. The resulting aggregation was less than that obtained from the adrenaline-treated platelets. This last observation is compatible with the suggestion that adrenaline brings about the conversion of intrinsic adenosine triphosphate in the platelet to diphosphate.

The following experiment shows that treated platelets that will not aggregate when diphosphate is added will still respond normally to adrenaline. Thus adrenaline-induced aggregation may not be caused by the liberation of diphosphate. Diphosphate, $0.1 \mathrm{ml}$. of $5 \mathrm{M} \times 10^{-3}$, was added at the same time

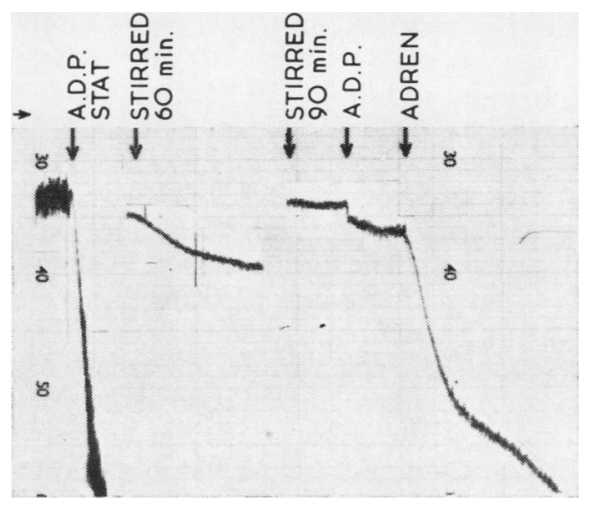

FIG. 4. Adenosine diphosphate $0.1 \mathrm{ml} . \mathrm{M} \times 10^{-2}$ was added simultaneously to three cuvettes containing $2 \mathrm{ml}$. platelet-rich plasma. The first cuvette was stirred at once; the second after incubating unstirred for 60 minutes, the third after 90 minutes. More diphosphate was then added to show the platelets could not aggregate with diphosphate. Adrenaline was then added and caused normal aggregation. to each of three cuvettes containing $2 \mathrm{ml}$. of citrated platelet-rich plasma. The first was stirred at once and showed immediate gross aggregation (Fig. 4). The second cuvette was not stirred till 60 minutes later when slight aggregation only was recorded. The third cuvette stirred after 90 minutes' stationary incubation showed no aggregation. Then a further $0.1 \mathrm{ml}$. of diphosphate was added and the stirring continued. Since no aggregation occurred, this confirmed that the platelets were almost completely unresponsive to the extrinsic diphosphate. Strong adrenaline was then added and gave a normal response. It was also shown that platelets incubated but not stirred with adrenaline finally became nonsticky. If diphosphate was then added, normal aggregation occurred (Fig. 5). These two experiments show that platelets incubated with either adrenaline or diphosphate lose their stickiness even though an excess of the compound is present, and that such platelets are still capable of becoming sticky again when the other compound is added. Thus, the production of stickiness by each of these compounds may be a different and independent process.

To test the effect of the inhibitors in vitro of aggregation on phenomena in vivo some compounds were injected intradermally and bleeding times carried out on the injection sites. The results are summarized in Table IV.

\section{DISCUSSION}

The problem may now be considered of how these many different compounds produce the same remark-

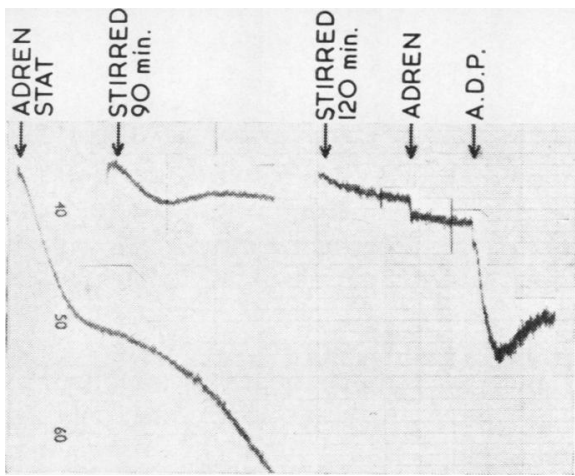

FIG. 5. Adrenaline $0 \cdot 1$ of $M \times 10^{-2}$ was added simultaneously to three cuvettes of platelet-rich plasma. The first cuvette was stirred at once. The second, after incubating unstirred for 90 minutes, was then stirred. The third was stirred after 120 minutes. More adrenaline was added to show the platelets could not aggregate with adrenaline. Diphosphate was then added and caused normal aggregation. 
TABLE IV

\begin{tabular}{lccc}
\multicolumn{4}{c}{ EFFECT OF INHIBITORS, ETC., ON BLEEDING TIME } \\
$\begin{array}{l}\text { Material } \\
\text { Injected }\end{array}$ & $\begin{array}{l}\text { Amount Injected } \\
\text { (mg.) }\end{array}$ & $\begin{array}{l}\text { No. of } \\
\text { Observations }\end{array}$ & $\begin{array}{l}\text { Mean Bleeding } \\
\text { Time }(\text { min. })\end{array}$ \\
\hline Saline & & 8 & 3.5 \\
Adenosine & 0.25 & 4 & 4.5 \\
U.M.L. 491 & 0.1 & 4 & 3.95 \\
Phentolamine & 0.5 & 8 & $>12.5$ \\
Phentolamine & 0.05 & 4 & 5.5 \\
Dibenyline & 0.3 & 2 & 4.2 \\
Histamine & 0.05 & 12 & 3.75 \\
Hyaluronidase & $301 . \mathrm{V}$. & 2 & 2.7
\end{tabular}

able end effect, namely aggregation. There is good evidence (Grette, 1962) that thrombin causes platelet triphosphate to be converted to diphosphate which is liberated. Indirect evidence has already been presented (O'Brien, 1963a) that tri-ethyl tin may cause the liberation of diphosphate. In this paper it has been shown that adrenaline and noradrenaline may liberate more diphosphate than was liberated by fragmenting the platelets. This again suggests that triphosphate may be converted to diphosphate and liberated. Recently Hovig (1963) has shown that collagen also causes the release of diphosphate. There is no evidence whether or not 5-hydroxytryptamine liberates diphosphate although it is known that triphosphate is involved in its uptake into the platelet (Born, Ingram, and Stacey, 1958). Thus it appears that following all additions which cause aggregation, diphosphate is liberated. Added diphosphate itself causes aggregation and adenosine and the monophosphate inhibit the effects of added diphosphate (Born and Cross, 1963a; O'Brien, 1963c) and of adrenaline etc. Therefore, it is possible that diphosphate is the final common cause of aggregation if such exists as already suggested by Clayton and Cross (1963). The appearance of diphosphate could, however, be a coincidental occurrence. If diphosphate brings about aggregation when any of these compounds is added, then platelets, however treated, must respond to added diphosphate if they can still respond to the other compounds. In fact it was shown that on incubating platelet-rich plasma, the disorganization of the platelets with time affected the response to diphosphate last. Since aggregation caused by added diphosphate is inhibited by triphosphate, it may be asked why added triphosphate enhances aggregation caused by adrenaline and speeds up aggregation caused by tri-ethyl tin. In the presence of adrenaline and tri-ethyl tin, the concentration of triphosphate in the plasma may fall below the inhibitory concentration; or an increased amount of intrinsically formed diphosphate may be in a preferred situation inaccessible to extrinsic triphosphate. In any case the effects of 'triphosphate' solutions are likely to be complex; the increased aggregating acti- vity noted when triphosphate solutions are stored would appear to be due to the production of some diphosphate.

How all these compounds apparently bring abog the mobilization of diphosphate is unclear, but evidently the pathways are different, as the details the aggregation and the inhibitors indicate. (T synergistic effects also suggest different and compementary pathways.) Adenosine diphosphate causes immediate aggregation and a second small dose causes reaggregation. Thrombin has no effect for 10 seconds and thereafter the pattern is the same $\overrightarrow{c s}$ for diphosphate. A second dose of 5-hydrox 4 tryptamine is inactive. Adrenaline produces two waves of aggregation which probably indicate t $\overline{\mathrm{w} p}$ different processes. This latter finding is reminiscent of Bülbring's suggestion (1960) of the effects Jf adrenaline on a muscle cell, the first an immediafte one on the membrane affecting its permeability amd the second a delayed metabolic one. Collagen pröduces its effect only after 55 to 65 seconds (Hovs, 1963). That the effects of adrenaline and 5-hydroxytryptamine are mediated through specific receptor sites is emphasized by the specific inhibition of each by the anti-adrenaline drug phentolamine and by the anti-serotonins Ro 3/0837 and UML 491 respective .

Mitchell and Sharp (1964) suggested that concentration gradient of an appropriate compougd across some membrane might be responsible for 'stickiness'. The following observations are com patible with this suggestion: platelets incubated with an excess of diphosphate or 5-hydroxytryptamine or adrenaline are initially sticky and subsequently loc their stickiness. Platelets incubated with excess diphosphate or adrenaline till they have lost their stickiness will aggregate normally on the addition if other compounds. However, 5-hydroxytryptaminge (Born, Ingram, and Stacey, 1958; Sano, Kakimotô, and Taniguchi, 1958) and adrenaline (Born, Horn kiewicz, and Stafford, 1958) are known to be activedy transported into the platelet and these transpost systems probably involve high energy phosphate and a triphosphatase. The inactivation of added diphos phate by the platelets may also involve a triphosphatase. It is therefore possible to suggest that thes three compounds which cause immediate aggreges tion do so by stimulating independently an adenosige tri-phosphatase and not by liberating diphosphate.

The three compounds that cause aggregation aft a delay, namely, thrombin ( $10 \mathrm{sec}$.), collagen ( $60 \mathrm{sec}$, and tri-ethyl tin $(300 \mathrm{sec}$.), must each have a different action, but in all three situations a triphosphatase $\overline{\Phi s}$ presumably stimulated since diphosphate is liberate Tri-ethyl tin in another situation is known to stim late a triphosphatase (Aldridge, 1958). Thus plateleft aggregation caused by adenosine diphosphate, 


\section{Outside \\ Platelet Membrane Inside}

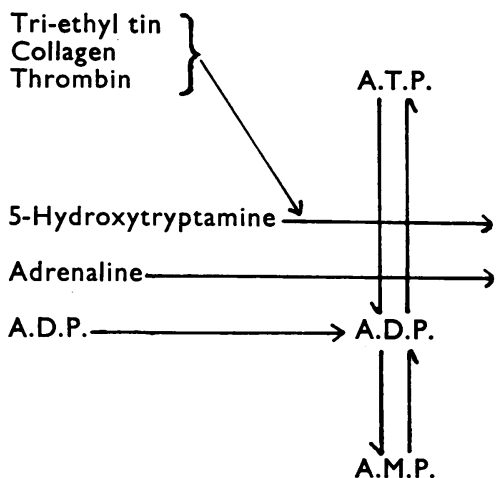

FIG. 6. A hypothetical scheme of the sites of action of the different aggregating agents.

adrenaline, noradrenaline, 5-hydroxytryptamine, thrombin, collagen, and tri-ethyl tin might be due to the stimulation of an adenosine triphosphatase. This tentative suggestion is represented diagrammatically in Figure 6. The formation of, or the presence of added, diphosphate might equally be the final common cause of aggregation but this seems less likely in view of the experiments summarized in Figures 4 and 5 . The effects of the specific anti-adrenaline and anti-serotonin inhibitors, of adenosine, the mono- and tri-phosphates, and of the non-specific inhibitors like cocaine (antiadhesive compounds) are compatible with either hypothesis.

The observation that an anti-adrenaline compound prolongs the bleeding time suggests but does not prove that adrenaline (or noradrenaline) plays a part in haemostasis, and if so, it would seem reasonable to suggest that adrenaline causes platelet aggregation and/or increased stickiness to the damaged vessel wall. This suggestion gains support from the observation that another anti-adrenaline compound, dibenyline, does not (except at high concentration) block adrenaline-induced aggregation in vitro and it does not prolong the bleeding time. It is of interest that Derouaux (1941) showed that adrenaline in some circumstances shortens the bleeding time in rabbits and that Ozge, Mustard, Hegardt, Rowsell, and Downie (1963) claim that adrenaline shortens the platelet half life in pigs.

There is a remarkable similarity in the response of three catecholamines and two inhibitors of adrenaline in three situations, namely, the stimulation of smooth muscle of the nictitating membrane of the cat, the effect in vitro on platelet aggregation, and the effect in vivo on the bleeding time, which presumably reflects an effect on aggregation in vivo (Table $\mathrm{V}$ ). Accordingly adrenaline may have some common basic mechanism in these three different biological situations.

TABLE V

SOME EFFECTS OF THE CATECHOLAMINES AND THEIR INHIBITORS

\begin{tabular}{|c|c|c|c|}
\hline & $\begin{array}{l}\text { Smooth } \\
\text { Muscle } \\
\text { Stimulated }\end{array}$ & $\begin{array}{l}\text { Platelet } \\
\text { Aggregation } \\
\text { in Vitro }\end{array}$ & $\begin{array}{l}\text { Bleeding Time } \\
\text { Prolonged }\end{array}$ \\
\hline $\begin{array}{l}\text { Adrenaline } \\
\text { Noradrenaline } \\
\text { Isoprenaline }\end{array}$ & $\begin{array}{l}\text { Yes } \\
\text { Weaker } \\
\text { No }\end{array}$ & $\begin{array}{l}\text { Yes } \\
\text { Weaker } \\
\text { No }\end{array}$ & - \\
\hline \multicolumn{4}{|c|}{ Adrenaline Effect Inhibited by: } \\
\hline $\begin{array}{l}\text { Phentolamine } \\
\text { Dibenylene }\end{array}$ & $\begin{array}{l}\text { Yes } \\
\text { No }\end{array}$ & $\begin{array}{l}\text { Yes } \\
\text { No }\end{array}$ & $\begin{array}{l}\text { Yes } \\
\text { No }\end{array}$ \\
\hline
\end{tabular}

This work was carried out during the tenure of a grant from the Wessex Regional Hospital Board which is gratefully acknowledged. It is a pleasure to acknowledge the careful technical assistance of Miss J. M. Patrick, B.Sc. I have received helpful suggestions from Dr. R. G. Macfarlane, F.R.S., Dr. J. Marks, and Professor W. D. Paton, F.R.S. whom I would like to thank.

The nucleotides were obtained from Sigma Chemicals, the adrenaline acid tartrate from British Drug Houses, the noradrenaline (Levophed) and its solvent from Bayer Products. Serotonin and Ro 3/0837 were given by Roche Products, dibenyline by Smith Kline and French and UML 491 (Deseryl) by Sandoz Products. Dr. W. H. Aldridge kindly gave the tri-ethyl tin.

\section{REFERENCES}

Aldridge, W. N. (1958). Biochem. J., 69, 367.

Borchgrevink, C. F. (1960). Acta med. scand., 168, 157.

Born, G. V. R., and Cross, M. J. (1963a). J. Physiol. (Lond.), 166, 29P. - - (1963b). Ibid, 168, 178.

Hornykiewicz, O., and Stafford, A. (1958). Brit. J. Pharmacol., 13, 411.

_, Ingram, G. I. C., and Stacey, R. S. (1958). Ibid., 13, 62.

Bülbring, E. (1960). In Adrenergic Mechanisms. A Ciba Foundation Symposium, p. 275. Churchill, London.

Clayton, S., and Cross, M. J. (1963). J. Physiol., 169, 82P.

Derouaux, G. (1941). Arch. int. Pharmacodyn., 65, 125.

Gaarder, A., Jonsen, J., Laland, S., Hellem, A., and Owren, P. A. (1961). Nature (Lond.), 192, 531.

Grette, K. (1962). Acta physiol. scand., 56, suppl. 195.

Hovig, T. (1963). Thrombos. Diathes, haemorrh. (Stuttg.), 9, 264.

Mitchell, J. R. A., and Sharp, A. A. (1964). Brit. J. Haemat., 10, 78. O'Brien, J. R. (1962). J. clin. Path., 15, 446.

- (1963a). Thrombos. Diathes. haemorrh. (Stuttg.), 9, 120.

- (1963b). J. Atheroscler. Res., 3, 262.

(1963c). J. clin. Path., 16, 223.

Ozge, A. H., Mustard, J. F., Hegardt, B., Rowsell, H. C., and Downie, H. G. (1963). Canad. med. Ass. J., 88, 265.

Sano, I., Kakimoto, Y., and Taniguchi, K. (1958). Amer. J. Physiol., 195, 495. 